\title{
IMAGE RESTORATION WITH OPERATORS MODELED BY ARTIFICIAL NEURAL NETWORKS
}

\author{
Ana Paula Abrantes de Castro e José Demisio Simões da Silva \\ Instituto Nacional de Pesquisas Espaciais - INPE, São José dos Campos, SP, Brasil \\ [apaula,demisio]@lac.inpe.br \\ Elcio Hideiti Shiguemori \\ Instituto de Estudos Avançados - IEAv, São José dos Campos, SP, Brasil \\ elcio@ieav.cta.br
}

\begin{abstract}
This paper presents a new approach to image restoration based on $A N N$, considering the learning of the inverse process using a standard image for training under a multiscale approach. Different models of ANN were tested and compared with the traditional techniques. The standard image was artificially degraded to simulate some types of frequent degradation problems. Due to the huge amount of data generated for training the ANN, this paper uses clustering techniques to reduce the training set. The paper proposes a simple restoration method that leads to a sub-optimal solution without the need of prior knowledge estimation of the degradation phenomenon. The ANN based filters were tested with different kinds of degraded images. The mean squared error and the signal-to-noise ratio were used as performance indices to measure the quality of the results of the ANN and of some of the existing methods for comparison. The results show that the ANN based restoration algorithms as proposed in this paper are effective restoration methods. The main advantage of the proposed approach is related to the fact that it does not require an estimation of prior knowledge of the degradation causes for each image.
\end{abstract}

\section{Introduction}

Due to the computational and technological development the use of digital images has considerably grown in the past years in several application fields as: security, monitoring, medicine, biology, industrial automation, astronomy, military, remote sensing, and autonomous navigation. These applications require computational methods of Digital Image Processing (DIP) to improve image quality to facilitate human perception and interpretation with the help of automatic machines.
Different approaches have been designed to restore images from their degraded versions. The methods try to compensate the possible existing problems of imaging systems, by assuming that acquisition systems may produce degraded images due to motion blur, atmospheric turbulence, and optical diffraction [13]. In addition, the methods assume that Gaussian-like distribution noise best models the kind of noise that may be found in acquired images [9]. Thus, designing restoration techniques is very important for image-based measurement systems since noisy and degraded images may significantly decrease the accuracy of feature extraction and object recognition operations.

Different methods for image restoration have been proposed, including the inverse filter, the Wiener filter, the moving-average filter, the parametric Wiener filter, mean-squared-error filters, the singular-value decomposition technique, deconvolution, and the bandpass filter [8], as well as, the regularization filter [2]. All of these methods characterize the imaging systems by their corresponding model.

The restoration of images is an inverse problem in which the real image has to be recovered from its probably degraded version. The methods usually require a priori knowledge of the degradation process to specify the input parameters for the inverse restoration approach [11]. Attempts to enhance the decision process in image restoration have led to modifications in the classic filters and some of the image restoration techniques have been modified to improve the results and reduce the computational complexity $[10,6,4,3,16]$.

In $[20,12,18,19,7,14]$ Artificial neural networks (ANN) have been used to restore images. [20] has designed a Hopfield network, whereas [12] has trained a six-layer feed-forward network based on the singular value decomposition technique. The work of [18] 
has proposed two approaches for the image restoration problem: a modified Hopfield neural network to implement a harmonic model, and a variational partial differential equations based algorithm. An impulse noise filtering operator algorithm has been proposed by [19], combining four center-weighted median filters with a simple adaptive neuro-fuzzy inference system. [7] has proposed a two stage based method involving the estimation of the blur function parameters that depends on the image degradation model and the reconstruction of the blurred images using a Hopfield recurrent neural network. The work of [14] has proposed a Genetic Algorithm-based Fuzzy image filter to remove additive identical independent distribution impulse noise from highly corrupted images. It consists of a fuzzy logic based reconstruction process, a fuzzy filtering process, a genetic learning process, and an image knowledge base.

The ANN models have been employed in image restoration; however, in most of works, the training and generalization tests have been applied to different regions of the same image. For instance, in [1] a MLP neural network was applied to reduce the blurring in degraded gray level images corrupted by addictive noise. The MLP neural network was trained with the degraded image as input and the original image as output. In [17] the training data set is composed by the degraded image and the correlation between the original image and the degraded image. In [15] an MLP was trained with a fuzzy logic filter as input and the original image as the desired output.

In this paper we present a novel ANN based multiscale restoration approach, considering the inverse process of learning using a standard type of image. The differences of the proposed technique are the use of a generic degraded image model to train the proposed neural networks and the use of a multiscale like approach to explore the influence of the neighborhood of a pixel in the restoration process, as well as, the independence of the estimation of prior knowledge of the degradation process for the restoration. Thus, a main advantage of the proposed method is the possibility of using the same filter in images from different sensors avoiding the estimation of a priori knowledge of the image and the possible noise present.

The method employs different models of neural networks, as alternatives to the process of image restoration. The networks used were: a Multi Layered Perceptron (MLP), a modified Radial Basis Functions (RBF), and a Hopfield neural network (HNN). The performances of the proposed neural network based filters were compared with those of existing techniques reported in existing literature.
In the results presented in the paper, the degradation effects are simulated by applying the degradation model in [8]. In this model, the image is first convoluted with a low-pass Gaussian filtering and then noise is added to the resulting image to simulate interference, transmission errors, etc. The noise rate adopted is assumed to be commonly found in digital images. Thus, in the supervised training process the degraded image data is provided as input to the ANN and the nondegraded image pixel is presented as the corresponding output.

The main difference of the present approach to existing ones relies on the use of space relations taken from the surroundings of the considered pixel in different scales, which makes it possible for the ANN to capture existing space relations among the considered pixels in the image. This approach attempts at coming up with a simple method that leads to an optimum solution to the problem without the need to obtain or estimate a priori knowledge of possible existing degradations in the images.

In trying to generalize the results obtained, the trained ANNs were submitted to indoor, outdoor, and satellite artificially degraded images to verify their (or validation) performances on different image types. The results are compared to existing restoration approaches (especially the Wiener filter).

The paper is organized as follows. Section 2 brings some basic definitions of the restoration problem. Section 3 describes the proposed restoration method. Section 4 presents some experimental results, with a quantitative analysis. Finally, Section 5 brings the conclusion for the work.

\section{Image restoration}

In general, image restoration techniques are oriented by the degradation model and consist of the application of an inverse process to obtain the original image $[8,2]$ based on a priori knowledge of the degradation phenomenon.

The degraded model in the spatial domain is represented by Equation 1 in which $f(x, y)$ is the original image and $g(x, y)$ the degraded one. In the model, $\eta(x, y)$ represents an additive noise introduced by the system, and $h(x, y)$ is the point spread function of the blurring degradation component, which may be simulated by a low-pass Gaussian filter. The same model in the frequency domain by the Fourier Transform is given by Equation 2 [8].

$$
\begin{gathered}
g(x, y)=h(x, y) * f(x, y)+\eta(x, y) \\
G(u, v)=H(u, v) \cdot F(u, v)+N(u, v)
\end{gathered}
$$


A common method used in image processing is the Wiener filter. A priori estimate of the original image frequency spectrum $F(u, v)$ can be obtained, given knowledge of the degradation process. The Wiener filter operation is described by Equation 3 .

$$
\hat{F}(u, v)=\left[\frac{1}{H(u, v)} \cdot \frac{|H(u, v)|^{2}}{|H(u, v)|^{2}+S_{\eta}(u, v) / S_{f}(u, v)}\right] G(u, v)
$$

where $|H(u, v)|^{2}$ is the square magnitude of the frequency spectrum of the degradation model, $S_{\eta}(u, v)$ and $S_{f}(u, v)$ are the average square magnitude frequency spectrums of the noisy and the original images, respectively. The ratio $S_{\eta}(u, v) / S_{f}(u, v)$ is called the noise-to-signal power ratio.

\section{The Proposed Approach}

In this paper an ANN based multiscale image restoration approach is proposed. The calibration of the method requires two distinct phases: in the first a modified Kohonen neural network, using a growing strategy based on a similarity threshold level is employed to cluster the training data set; in the second phase an MLP, an RBF, and a Hopfield are trained and employed to recover the inverse reconstruction model.

The three neural networks are trained with the data acquired from the artificial degraded image of gray level co-centered circles. This training strategy attempts at making the ANN learn embedded space relations between a degraded pixel and its neighborhood in order to be associated to its non-degraded version. The flowchart diagram in Figure 1 represents the main process of the proposed approach.

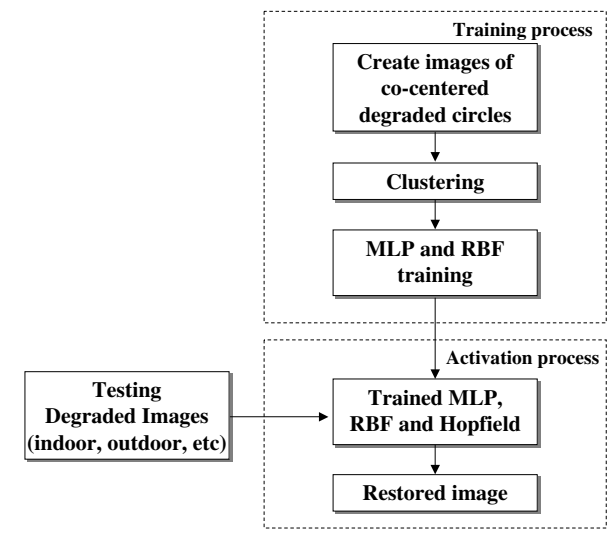

\section{Figure 1. Flowchart diagram of the proposed approach}

Figure 1 shows the two main processes of the proposed method. The training process consists of building the training sets by creating the synthetic degraded image. Due to the large sizes of the sets obtained, the images are then presented to a Kohonen based clustering algorithm in order to produce reduced sets for training purposes. In the activation process the available testing images are first artificially degraded and then they are submitted to trained neural networks for restoration and performance analysis.

\subsection{Training data}

The proposed approach follows the assumption that the effects of the degradation sources mentioned before are commonly found in images. Thus, the 8-bit degraded gray level image of co-centered circles was assumed to represent all possible common interferences in an image, independent of the source of the image. Figure 2.a shows the degraded image of the co-centered circles on the left and the corresponding non-degraded version on the right.

The multiscale approach is achieved by sequentially extracting $3 \times 3,5 \times 5,7 \times 7$ windows around a pixel in the degraded version of the image, which is then associated to the corresponding pixel at the center position of the a $7 \times 7$ window in the original non-degraded image.

Effectively, a $7 \times 7$ window is extracted and then, a $3 \times 3$ and a $5 \times 5$ windows are derived by subsampling the extracted $7 \times 7$ window. In addition, the $5 \times 5$ and $7 \times 7$ windows are subsampled to form $3 \times 3$ windows. Then, the process results in 3 windows of $3 \times 3$ pixels. Each of these windows is rearranged to form $9 \mathrm{x} 1$ vectors. The resulting vectors are finally assembled in one $27 \times 1$ vector that is added to the set of training vectors. Such process has been called in this paper as 'linearization'. Such vector is associated to the non-degraded pixel at the center of the equivalent $7 \times 7$ window in the original image. The process yield for each pixel a $27 \mathrm{x} 1$ vector (see Figure 2.b).
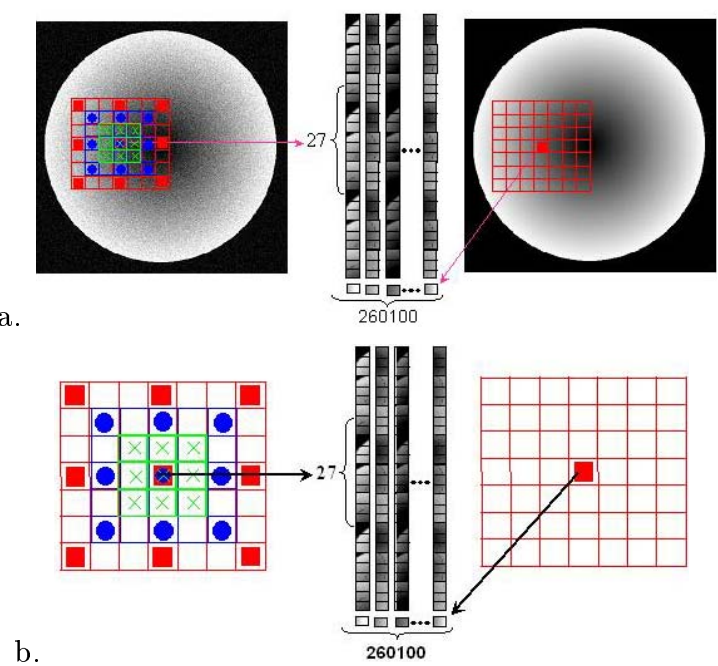

Figure 2. Multiscale approach: a) over all view of "linearization" in process and b) illustration of the $7 \times 7$ window neighborhood subsample method. 
The previous data assembling process results in a very large training data set that may take a long time for the MLP training phase. Thus, the previously cited Kohonen neural network is applied, in a data mining like approach, to reduce the amount of data for training purposes. Vector similarity thresholds are established to extract reduced sets of the original data. The experiments reported in this paper used a similarity threshold of 0.8 . However, the image data was submitted to 0.8 and 0.9 similarity thresholds. Table 1 shows the size of the resulting data sets obtained by the Kohonen network for dimension reduction employed in the training phase.

Table 1. Training set reduction

\begin{tabular}{|c|c|c|c|}
\hline Image & Size Image & Similarity & Vector Size \\
\hline Circle & $506 \times 506$ & 1.0 & 256036 \\
\hline Circle & $506 \times 506$ & 0.9 & 11318 \\
\hline Circle & $506 \times 506$ & 0.8 & 2886 \\
\hline
\end{tabular}

\subsection{Training process}

The MLP training process consisted of the simultaneous submission of the degraded image data as inputs and the pixel at the center of the corresponding $7 \times 7$ window in the non-degraded image as the corresponding output. The proposed MLP was designed with 1 hidden layer with 28 neurons and 1 output. However, different MLP architectures were tried. The logistic sigmoidal activation function was used for all the neurons. The trained MLP was then generalized by submitting different images to it.

To use the RBF network in the construction of a filter for image restoration required two changes in the methodology of constructing RBF networks. The first was in the architecture of the network with the inclusion of an extra hidden layer. The second change was in the form of setting the centers of the radial basis functions of the problem at hand. The first layer of hidden neurons form a base of 256 Gaussian functions, with $\sigma=1$. In the second layer neurons the logistic sigmoidal function was used as the activation function. The centers of the Gaussian functions of the first hidden layer were 256 vectors obtained from the training set used in training the MLP network. The original set with 2886 vectors (after clustering) was reclustered to result in a reduced set with 256 vectors. Chosen the centers of the first hidden layer, the training of the second hidden layer and output layer employs the error backpropagation algorithm to train the MLP. The algorithm ran iteratively for 100 times of training. The weights obtained were subjected to a generalization test on a degraded image test. The resulting restored image is subjected to the calculation of the signal/noise ratio which is then compared to the value obtained with the Wiener filter, considered to be the best among the traditional methods used in this work. The criterion for stopping the algorithm consisted of finding a value of the signal/noise ratio, for the RBF network, that as at least $5 \%$ higher than the value of the signal noise ratio obtained in the restoration by Wiener filter.

The use of the Hopfield network in this work follows the general ideas of this network as an associative memory, with the following changes: 1) the vectors are stored as fundamental memories vectors with discrete components assuming values between 0 and 255; 2 ) the activation function used is the hyperbolic tangent function, and 3) the update of the states of the neurons is synchronous. This assembly used the 256 vectors resulting from the reclustering of the $2886 \mathrm{vec}-$ tors generated by the multiscale approach. The activation of the network consisted of the presentation of $7 \times 7$ data extracted from the windows in the degraded image test. The state of the neurons of the Hopfield network were updated for a number of iterations or until there were not any significant change (within certain limits of tolerance) in the energy of the network given by the Liapnunov function $(E)$ expressed in Equation 4 .

$$
E=-\frac{1}{2} \sum_{i \neq j} \sum_{j} w_{i j} y_{j} y_{i}-\sum_{i} x_{i} y_{i}+\sum_{i} b_{i} y_{i}
$$

During generalization, the ANN is exposed to a set of different images artificially degraded with the same parameters of Equation 1.

\section{Experiments}

In this section, we present results of some of the experiments conducted in which the proposed technique was used to restore degraded images. The trained neural network architecture was used to restore artificially degraded versions of different images: the Lenna image commonly used in the computer vision literature, and outdoor images, such as, satellite images of different applications. Such images were submitted to the same degradation process used in the construction of the training image. The use of different image data aims at verifying the adequacy and robustness of the present approach for the image restoration problem as stated in [8]. In [5], this same methodology was applied in tomography brain images degraded by the 
capture system, and the neural restoration system improved the classification process, showing the adequacy of the method.

Our method was applied for 7 images and the results, for 3 of them (one of each type) can be observed in the Figures 3, 4 and 5. A comparison of the proposed method and the Wiener filter is performed through a quantitative analysis by calculating the Mean Square Error (MSE) and the Signal-to-Noise-Ratio (SNR). Tables 2 and 3 show the qualitative analysis for all the experiments.

Figures 3, 4 and 5 present, respectively, the Lenna, the LANDSAT (LS) 1 and the CBERS (CB) 2 images: a) without noise; b) artificially degraded by a 3rd Gaussian blurring filter with (Gauss $\sigma^{2}=10$ ) and additive noise with (mean) $\mu=0$ and (variance) $v=0.01$, c) MLP (MLP08) restoration result with similarity $=0.8, \mathrm{~d})$ MLP $($ MLP09) restoration result with similarity $=0.9$, e) $\mathrm{RBF}$ restoration result; and $\mathrm{f}$ ) Hopfield (Hopf) restoration result.
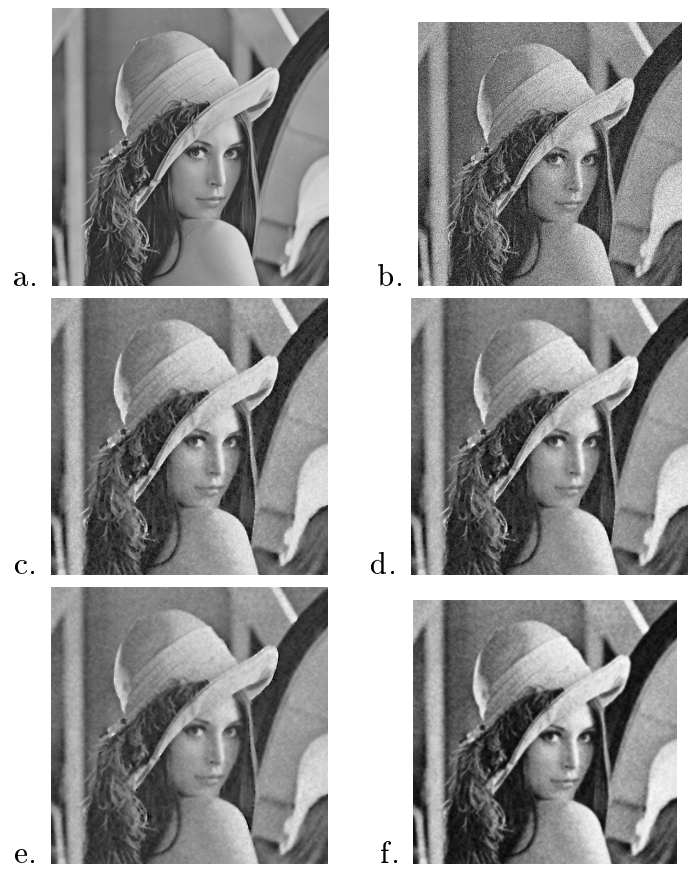

Figure 3. Generalization tests with Lenna image: a) without noise; b) degraded; c) MLP08; d) MLP09; e) RBF; and f) Hopf.

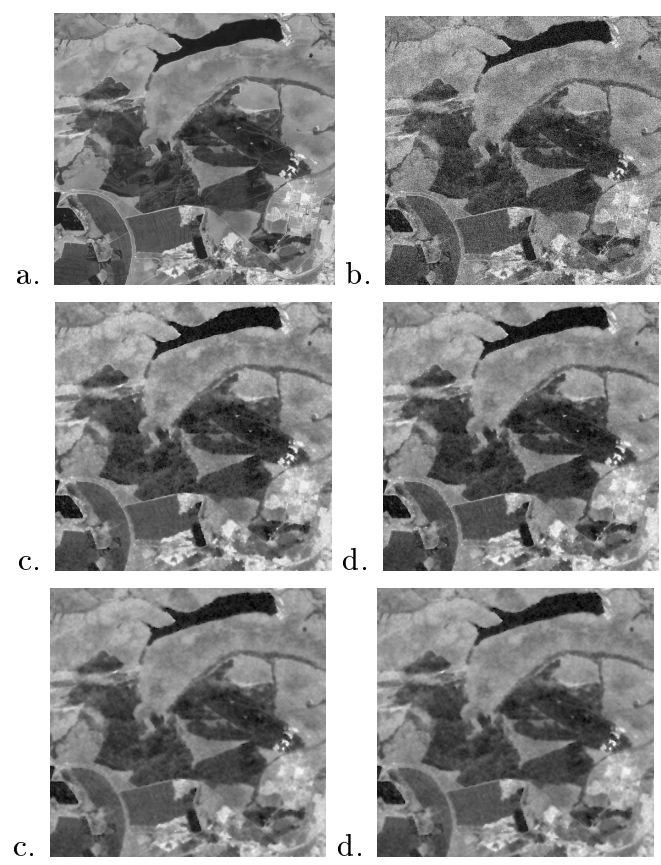

Figure 4. Generalization tests with LandSat 2 image: a) without noise; b) degraded; c) MLP08; d) MLP09; e) RBF; and f) Hopf.

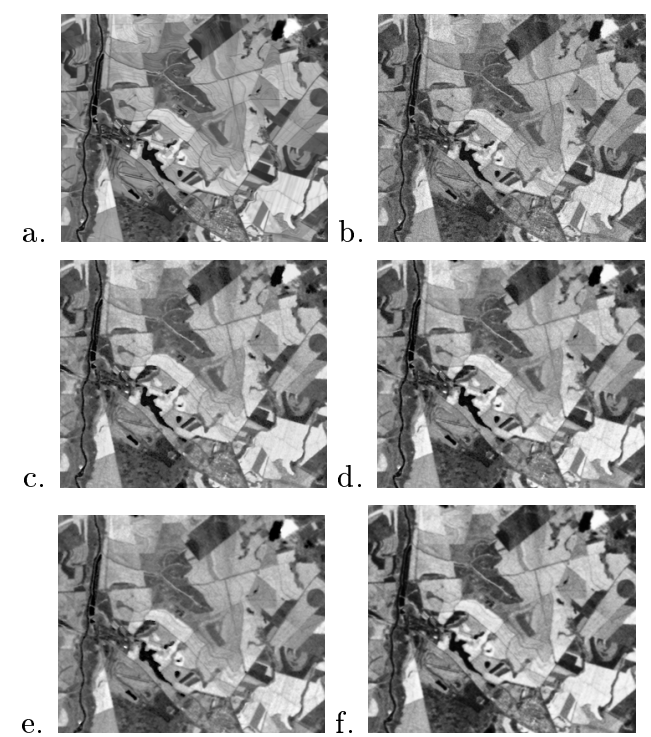

Figure 5. Generalization tests with Cbers 1 image: a) without noise; b) degraded; c) MLP08; d) MLP09; e) RBF; and f) Hopf.

The obtained values for the MSE and the SNR between the original image and the degraded version, and between the original and restored images are presented in Table 2 and 3, respectively. The first col- 
umn refers to the image employed in the tests (Lenna, LandSat1 (LS1), LandSat2 (LS2), LandSat3 (LS3), CBERS1 (CB1), CBERS2 (CB2), CBERS3 (CB3)), the second column to the degraded images (D), the third column refers to the restoration results obtained by Wiener filter (WF), the fourth column refers to the restoration results obtained by multilayer perceptron with similarity $=0.8$ (MLP08), the fifth column to multilayer perceptron with similarity $=0.9$ (MLP09), the sixth column refers to the radial Basis Function network (RBF) results, and the seventh column refers to the Hopfield network (Hopf) results.

Table 2. Mean Square Error (MSE).

\begin{tabular}{|c|c|c|c|c|c|c|}
\hline Image & D & WF & $\begin{array}{c}\text { MLP } \\
0.8\end{array}$ & $\begin{array}{c}\text { MLP } \\
0.9\end{array}$ & RBF & Hopf \\
\hline Lenna & 25.79 & 11.14 & 9.81 & 10.32 & 10.12 & 10.19 \\
\hline LS1 & 34.40 & 10.79 & 7.75 & 8.66 & 8.59 & 11.80 \\
\hline LS2 & 25.35 & 11.48 & 10.82 & 11.07 & 11.39 & 11.47 \\
\hline LS3 & 37.55 & 11.78 & 11.87 & 12.21 & 14.12 & 15.38 \\
\hline CB1 & 26.55 & 11.05 & 9.78 & 10.29 & 9.66 & 10.53 \\
\hline CB2 & 91.94 & 12.39 & 12.13 & 11.54 & 13.35 & 18.89 \\
\hline CB3 & 49.62 & 11.35 & 10.95 & 11.11 & 12.27 & 13.47 \\
\hline
\end{tabular}

A better performance of the ANN restoration can be verified by comparing its smaller MSE and larger SNR, with the corresponding values obtained with the Wiener restoration filter. A smaller MSE means that the restored image is closer to the original version. The results obtained show that the approach is very promising. In Tables 2 and 3 we can verify that, compared to the Wiener Filter results, the ANN model presents better results for 6 of the 7 experiments performed, in which noise decreases. In a particular case, for the CBERS images, the ANN model performed better in 3 of the 3 images tested.

Table 3. Signal-to-Noise-Ratio (SNR).

\begin{tabular}{|c|c|c|c|c|c|c|}
\hline Image & D & WF & $\begin{array}{c}\text { MLP } \\
0.8\end{array}$ & $\begin{array}{c}\text { MLP } \\
0.9\end{array}$ & RBF & Hopf \\
\hline Lenna & 5.55 & 12.68 & 13.78 & 13.34 & 13.51 & 13.45 \\
\hline LS1 & 4.41 & 11.42 & 14.28 & 13.33 & 13.40 & 10.64 \\
\hline LS2 & 5.09 & 11.93 & 12.44 & 12.24 & 12.00 & 11.94 \\
\hline LS3 & 8.14 & 14.35 & 14.29 & 14.05 & 12.78 & 12.04 \\
\hline CB1 & 6.11 & 13.25 & 14.31 & 13.87 & 14.43 & 13.68 \\
\hline CB2 & 5.70 & 10.70 & 10.88 & 11.31 & 10.05 & 7.03 \\
\hline CB3 & 6.39 & 12.74 & 13.04 & 12.92 & 12.06 & 11.25 \\
\hline
\end{tabular}

\subsection{Additional experiments}

Additional experiments were carried out by varying the noise rate $(\mu=0$ and $v=0.01,0.05$ and 0.10$)$ and 3rd, 5th and 7th Gaussian blurring with (Gauss $\sigma^{2}=10$ ) artificially added to the image in the process of degradation.

\subsubsection{Lenna image with addtive noise}

Table 4 shows the results of images restoration with artificial noise only $\mu=0$ and $v=0.01,0.05$ and 0.10 .

Table 4. Results for Lena image degraded with noise $v=0.01, v=0.05, v=0.1$ e $\mu=0$

\begin{tabular}{|l|c|c|c|c|c|c|}
\hline \multirow{3}{*}{ Lena } & \multicolumn{2}{|c|}{$\begin{array}{c}v=0.01 \mathrm{e} \\
\mu=0\end{array}$} & \multicolumn{2}{c|}{$\begin{array}{c}v=0.05 \mathrm{e} \\
\mu=0\end{array}$} & \multicolumn{2}{c|}{$\begin{array}{c}v=0.1 \mathrm{e} \\
\mu=0\end{array}$} \\
\cline { 2 - 7 } & MSE & SNR & MSE & SNR & MSE & SNR \\
\hline D & 25.31 & 6.61 & 52.94 & 2.34 & 69.06 & 1.25 \\
\hline WF & 11.13 & 12.68 & 22.70 & 6.50 & 29.30 & 4.29 \\
\hline MLP08 & 9.80 & 13.79 & 19.46 & 7.83 & 28.27 & 4.59 \\
\hline MLP09 & 10.35 & 13.32 & 23.69 & 6.12 & 32.68 & 3.33 \\
\hline RBF & 10.18 & 13.46 & 15.05 & 10.06 & 19.12 & 7.96 \\
\hline Hopf & 10.08 & 13.54 & 17.84 & 8.59 & 33.50 & 3.12 \\
\hline
\end{tabular}

Through the Table 4 can be observed that the neural filters have better performance compared to the traditional filters. The filter MLP08 obtained the best result for the rate of noise $v=0.01$ and $\mu=0$ and for noise with $v=0.05, v=0.1$ and $\mu=0$ the filter that showed the best performance was RBF. This result can be observed through the values of SNR that were higher than other filters, as outlined in 4.

\subsubsection{Gaussian blurring and addtive noise}

Tables 5, 6 and 7 present the results of filters applied to Lenna image degraded with Gaussian blurring of 3rd, 5th and 7th order, respectively, with (Gauss $\sigma^{2}=10$ ) and additive noise varying $\mu=0$ and $v=0.01,0.05$ and 0.10 to test the ANNs approach.

Through Tables 5, 6 and 7 it can be observed that RBF neural filter sistematically present the better results. Through the analysis of these results it can be observed that the neural network performed better than the Wiener Filter (WF) for all of the noise levels used in the experiments. Thus, these results demonstrate the robustness of the methodology, when applied to images with different levels of noise. 
Table 5. Results for Lena image degraded with 3rd order Gaussian blurring.

\begin{tabular}{|l|c|c|c|c|c|c|}
\hline \multirow{3}{*}{ Lena } & \multicolumn{2}{|c|}{$\begin{array}{c}v=0.01 \mathrm{e} \\
\mu=0\end{array}$} & \multicolumn{2}{c|}{$\begin{array}{c}v=0.05 \mathrm{e} \\
\mu=0\end{array}$} & \multicolumn{2}{c|}{$\begin{array}{c}v=0.1 \mathrm{e} \\
\mu=0\end{array}$} \\
\cline { 2 - 7 } & MSE & SNR & MSE & SNR & MSE & SNR \\
\hline D & 25.90 & 6.26 & 53.48 & 2.19 & 69.45 & 1.16 \\
\hline WF & 12.22 & 11.88 & 23.44 & 6.22 & 29.85 & 4.12 \\
\hline MLP08 & 10,77 & 12,98 & 19,90 & 7.64 & 28,66 & 4.47 \\
\hline MLP09 & 11.07 & 12.73 & 24.04 & 5.99 & 33.10 & 3.22 \\
\hline RBF & 11.02 & 12.77 & 15.72 & 9.69 & 19.66 & 7.75 \\
\hline Hopf & 10.76 & 12.98 & 18.14 & 8.44 & 33.41 & 3.14 \\
\hline
\end{tabular}

Table 6. Results for Lena image degraded with 5th order Gaussian blurring.

\begin{tabular}{|l|c|c|c|c|c|c|}
\hline \multirow{2}{*}{ Lena } & \multicolumn{2}{|c|}{$\begin{array}{c}\boldsymbol{2}=01 \mathrm{e} \\
\mu=0\end{array}$} & \multicolumn{2}{c|}{$\begin{array}{c}v=05 \mathrm{e} \\
\mu=0\end{array}$} & \multicolumn{2}{c|}{$\begin{array}{c}v=1 \mathrm{e} \\
\mu=0\end{array}$} \\
\cline { 2 - 7 } & MSE & SNR & MSE & SNR & MSE & SNR \\
\hline D & 26.78 & 5.84 & 54.05 & 2.05 & 69.81 & 1.082 \\
\hline WF & 13.82 & 10.80 & 24.28 & 5.91 & 30.60 & 3.90 \\
\hline MLP08 & 12.17 & 11.90 & 20.74 & 7.27 & 29.25 & 4.29 \\
\hline MLP09 & 12.31 & 11.81 & 24.83 & 5.72 & 33.67 & 3.06 \\
\hline RBF & 12.09 & 11.96 & 16.55 & 9.24 & 20,30 & 7,47 \\
\hline Hopf & 11.99 & 12.04 & 18.79 & 8.13 & 33,57 & 3.098 \\
\hline
\end{tabular}

\section{Conclusion}

A neural network multiscale image restoration method was proposed for restoring degraded images based on an universal training data strategy. Experimental results show the adequacy of the proposed approach to solve the problem. Through a quantitative analysis it may be observed that the proposed method presents similar results to the ones obtained in the restoration process using the Wiener filter, reported in literature as the most used method for image restoration. As futures work, these results will be compared with more similar approaches: optimization approach and neighborhood pattern based method.

In most of the conducted experiments, a reduction of the degradation for the ANN restored images was performed as may be observed by the signal to noise ratio measurements.

An advantage of the proposed method is related to the fact a neural network approach may be less computationally expensive than the Wiener filter when dealing with very large image datasets, in addition to the easiness of implementation of the ANN models that may also be implemented directly imaging acquisition hardware.
Table 7. Results for Lena image degraded with 7th order Gaussian blurring.

\begin{tabular}{|l|c|c|c|c|c|c|}
\hline \multirow{2}{*}{ Lena } & \multicolumn{2}{|c|}{$v=0.01 \mathrm{e}$} & \multicolumn{2}{c|}{$v=0.05 \mathrm{e}$} & \multicolumn{2}{c|}{$v=0.1 \mathrm{e}$} \\
& \multicolumn{2}{|c|}{$\mu=0$} & \multicolumn{2}{c|}{$\mu=0$} & \multicolumn{2}{c|}{$\mu=0$} \\
\cline { 2 - 7 } & MSE & SNR & MSE & SNR & MSE & SNR \\
\hline D & 27.59 & 5.47 & 54.53 & 1.93 & 70.57 & 0.99 \\
\hline WF & 15.24 & 9.95 & 25.16 & 5.60 & 31.41 & 3.68 \\
\hline MLP08 & 13.51 & 11.01 & 21.41 & 7.00 & 30.00 & 4.07 \\
\hline MLP09 & 13.55 & 10.98 & 25.47 & 5.50 & 34.50 & 2.86 \\
\hline RBF & 13.22 & 11.19 & 17.34 & 8.84 & 21.22 & 7.08 \\
\hline Hopf & 13.20 & 11.20 & 19.51 & 7.81 & 34.55 & 2.85 \\
\hline
\end{tabular}

\section{References}

[1] K. Arakawa and H. Harashima. Neural implementation of arma type filters for image restoration. In Proceedings of 1990 IEEE International Conference on Communications, volume 2, pages 424-428, Atlanta, GA, USA, April 1990. IEEE International Conference on Communications - ICC'90, IEEE Computer Society.

[2] M. Bertero and P. Boccacci. Introduction to inverse problems in imaging (hardcover). Taylor and Francis Group, Philadelphia, England, 1 edition, 1998.

[3] F. M. Candocia and A. M. Díaz. A time-domain approach to determining inverse fir filters. In Proceedings of 2006 International Conference on Image Processing and Computer Vision \& Pattern Recognition., volume 1, pages 290-296, Nevada, USA, June 2006. International Conference on Image Processing and Computer Vision \& Pattern Recognition - IPCV 2006, CSREA Press.

[4] D. Cao and P. Guo. Blind image restoration based on wavelet analysis. In Proceedings of 2005 International Conference on Machine Learning and Cybernetics., volume 8, pages 4977-4982, Guangzhou, August 2005. International Conference on Machine Learning and Cybernetics - ICMLC 2005, IEEE Press.

[5] A. Castro, I. Drummond, and J. Silva. A multiscale neural network method for image restoration. TEMA - Tendências em Matemática Aplicada e Computacional, 9(1):41-50, 2008.

[6] J. Chen, J. Benesty, Y. Huang, and S. Doclo. New insights into the noise reduction wiener filter. IEEE Transactions on Audio, Speech and Language Processing, 14(4):1218-1234, July 2006.

[7] S.-Y. Fu, Y.-C. Zhang, L. Cheng, Z.-Z. Liang, Z.-G. Hou, and M. Tan. Motion based image deblur using recurrent neural network for power transmission line inspection robot. In Proceedings of 2006 International Joint Conference on Neural Networks., pages 3854-3859, Vancouver, July 2006. International Joint Conference on Neural Networks - IJCNN 2006, IEEE Computer Society. 
[8] R. C. Gonzalez and R. C. Woods. Digital image processing. Prentice Hall, New York, 2007.

[9] F. V. D. Heijden. Image based measurement systems: object recognition and parameter Estimation. John Wiley and Sons Inc, New York, NY, 2 edition, 1994.

[10] S. Jeon, G. Cho, Y. Huh, S. Jin, and J. Park. Determination of point spread function for a flat-panel $\mathrm{x}$-ray imager and its application in image restoration. Nuclear Instruments and Methods in Physics Research Section A: Accelerators, Spectrometers, Detectors and Associated Equipment, 563(1):167-171, July 2006.

[11] T. Kanungo and Z. Qigong. Estimating degradation model parameters using neighborhood pattern distributions: An optimization approach. IEEE Transactions on Pattern Analysis and Machine Intelligence, 26(4):520-524, Abril 2004.

[12] A. D. Kulkarni. Solving ill-posed problems with artificial neural networks. Neural Networks, 4(4):477-484, 1991.

[13] A. D. Kulkarni. Computer vision and fuzzy-neural systems. Prentice Hall, New Jersey, 1 edition, 2001.

[14] C.-S. Lee, S.-M. Guo, and C.-Y. Hsu. Genetic-based fuzzy image filter and its application to image processing. IEEE Transcations on Systems, Man, and Cybernetics - PartB: Cybernetics, 34(4):694-711, August 2005.

[15] L. Shutao, W. Yaonan, Z. Changfan, and M. Jianxu. Fuzzy filter based on neural network and its application to imagerestoration. In Proceedings of 2000 International Conference on Signal Processing., volume 2, pages 1133-1138, Beijing, August 2000. International Conference on Signal Processing- WCCC-ICSP 2000, IEEE Computer Society.

[16] F. Sroubek and J. Flusser. Multichannel blind deconvolution of spatially misaligned images. IEEE Transactions on Image Processing, 14(7):874-883, July 2005.

[17] A. Stajniak and J. Szostakowski. Neural implementation of arma type filters for image restoration. In Proceedings of 1995 International Conference on Image Processing., volume 2, pages 520-522, Washington, October 1995. International Conference on Image Processing - ICIP 1995, IEEE Computer Society.

[18] Y. D. Wu, Q. Z. Zhu, S. X. Sun, and H. Y. Zhang. Image restoration using variational pde-based neural network. Neurocomputing, 69(16-18):2364-2368, October 2006.

[19] M. E. Yüksel. A median/anfis filter for efficient restoration of digital images corrupted by impulse noise. International Journal of Electronics and Communications, 60(10):628-637, October 2006.

[20] Y. T. Zhou and R. Chellappa. Stereo matching using a neural network. In Proceeding of 1988 International Conference on Acoustics, Speech, and Signal Processing, volume 2, pages 940-943. International Conference on Acoustics, Speech, and Signal Processing ICASSP1988, IEEE Computer Society, April 1988. 